\title{
Spectral Intensities of Antiprotons and the Lifetime Of Cosmic Rays
}

\section{R. Cowsik ${ }^{* \dagger}$}

Physics Department and McDonnell Center for the Space Sciences

Washington University, St. Louis, MO 63130, USA E-mail: cowsik@wust 1. edu

\section{Tsitsi Madziwa-Nussinov}

Physics Department and McDonnell Center for the Space Sciences

Washington University, St. Louis, MO 63130, USA E-mail: cowsik@wust 1 . edu

\begin{abstract}
In this paper we note that the spectral intensities of antiprotons observed in Galactic cosmic rays in the energy range $\sim 1-100 \mathrm{GeV}$ by BESS, PAMELA and AMS instruments display nearly the same spectral shape as that generated by primary cosmic rays through their interaction with matter in the interstellar medium, without any significant modifications. More importantly, in the interstellar medium, with an assumed mean density, $\sim 0.5 \mathrm{~cm}^{-3}$, a constant residence time of $\sim$ $2.3 \pm 0.7$ million years, independent of the energy of cosmic rays, yields the observed intensities. A small additional component of secondary antiprotons in the energy below $10 \mathrm{GeV}$, generated in cocoon-like region of lumpy stellar debris surrounding the cosmic-ray sources, appears to be present. We discuss this result in the context of observations of other secondary components like positrons and Boron, and conclude with general remarks about the origins and propagation of cosmic rays.
\end{abstract}

The 34th International Cosmic Ray Conference,

30 July- 6 August, 2015

The Hague, The Netherlands

\footnotetext{
* Speaker.

${ }^{\dagger}$ Acknowledgements: It is a pleasure to thank M. H. Israel, W. R. Binns, P. Blasi and M. A. Lee for extensive discussions related to this paper.
} 


\section{Analysis of the spectral intensities of cosmic-ray antiprotons.}

The spectral intensities of antiprotons in cosmic rays, measured by the PAMELA [1], BESS [2] and AMS [3-5] instruments provide a crucial diagnostic tool to understand the origin and propagation of cosmic rays and complement the information obtained by studying the $\mathrm{B} / \mathrm{C}$ ratio, the spectrum of positrons, and the anisotropy in the intensity of cosmic rays. The main objective of this paper is to show that all these observations can be understood in a straightforward manner within the framework of the nested leaky box (NLB) model [6].

We display in Fig. 1, the observed spectral intensities of the antiprotons and in Fig. 2, the antiproton to proton ratio as a function of their kinetic energies. An inspection of these figures shows that at high energies the spectral index of antiprotons is nearly identical to that of the protons. Below $\sim 10 \mathrm{GeV}$ there is a small additional component in the antiproton flux. We interpret these aspects in the context of the NLB model and to this end we adopt the same features and the parameters of the NLB model that we used earlier to interpret the $\mathrm{B} / \mathrm{C}$ ratio, the positron spectral intensities and the positron fraction [7-8]. The main features of the model are given below:

1. Cosmic rays are accelerated in sources with a spectrum that is the same as that of the observed spectrum of the primary cosmic rays

$$
q_{p}(E) \approx q_{0} E^{-2.7}
$$

2. Each of the sources is surrounded by a lumpy shell of stellar debris. Subsequent to their generation, the cosmic rays are transported across such cocoons, in an energy-dependent fashion with higher energy particles leaking more rapidly into the general interstellar medium. For $T>1 \mathrm{GeV}$, the leakage lifetime is given by

$$
\tau_{c}(E) \approx \tau_{0} T^{(0.01-\zeta \ln T)}
$$

Here $\mathrm{T}$ is the kinetic energy per nucleon of the nuclei or per $e^{+}$or $e^{-}$, and $\zeta \approx 0.1$. Note that this energy dependence is nearly a power law that steepens with increasing energy. During this transport the cosmic rays suffer collisions with the material of the debris, generating secondary particles and radiation.

3. The transport of cosmic rays subsequent to their injection into the general interstellar medium is assumed to be independent of energy at least up to $100 \mathrm{TeV}$, beyond which it is expected to decrease with energy. Below this energy the leakage lifetime from the Galaxy is taken to be

$$
\tau_{G}=\text { constant }
$$

The kinematics of the production of $\bar{p}$ differs from that of the production of B-nuclei, which in turn is different from that of $e^{+}$. The threshold for the production of antiprotons is $7 m_{p} \approx 6.6 \mathrm{GeV}$ and even at threshold, the antiproton emerges from the collision with an energy of $2 m_{p} \approx 1.88 \mathrm{GeV}$ in the lab frame. As the primary energy increases, the emerging $\bar{p}$ spectrum spreads both towards the low and high energies, as shown in Fig. 3. There are several parameterizations of the Lorentz invariant product $E d^{3} \sigma / d p^{3}$, for the production of antiprotons in high energy collisions [9-10] 
and we have used the prescription by Kappl and Winkler [11] in these calculations. There are two contributions, one from the interactions in the stellar debris surrounding the sources, where the primary spectrum has a spectral intensity $F_{c} \sim E^{-2.7} T^{(0.01-\zeta \ln T)}$ and that in the interstellar medium with $F_{p} \sim E^{-2.7}$. These two contributions are shown in Fig. 1 as the chain-dotted line and the dashed line respectively. Note that their sum fits the observed spectrum well. The $\bar{p} / p$ ratio is also reproduced well, as shown in Fig. 2.

The grammage encountered by cosmic rays in the stellar debris surrounding the sources and in the general interstellar medium are estimated by fitting the measured $\mathrm{B} / \mathrm{C}$ ratio in cosmic rays. It may be noted by an inspection of Fig. 4 that around $\sim 1 \mathrm{GeV} /$ nucleon about $75 \%$ of the Boron nuclei are generated by spallation in the stellar debris and about $25 \%$ in the interstellar medium. As the energy increases the grammage in the shell of stellar debris decreases according to the energy dependence given in Eq. 2, whilst the interstellar contribution remains constant and dominates at the highest energies. The simple kinematics of the production of B-nuclei in spallation reactions of heavier nuclei makes this procedure transparent: The B nuclei emerge from the spallation reactions with almost exactly the same energy per nucleon as their parent nuclei, thereby establishing a one to one correspondence between the $\mathrm{B} / \mathrm{C}$ ratio and the grammage. The NLB fit to the $\mathrm{B} / \mathrm{C}$ ratio is shown in Fig. 4. The value of the residence time in the Galaxy thus derived corresponds to 2.3 Myr for an assumed mean density, $n_{H} \approx 0.5 \mathrm{~cm}^{-3}$.

The kinematics of the production of positrons is significantly more complicated: the first step is the production of positive pions that carry away typically $\sim 10 \%$ of the energy of the primary nucleons. The $\pi^{+}$decay into $\mu^{+}+v_{\mu}$ and subsequently $\mu^{+}$decays into $e^{+}+v_{e}+\bar{v}_{\mu}$. The positrons will thus have a broad spectrum and the weighted mean of their energy fraction is $\sim 5 \%$ of that of the primary. Similarly, $\bar{p}$ production also requires primary particles of considerably higher energy. Thus the parent particles of $e^{+}$and $\bar{p}$, mostly protons, leak rapidly away from the cocoon into the interstellar medium, where bulk of high-energy $\bar{p}$ and $e^{+}$are produced. Approximate scaling of the cross sections accords them the same spectra, $\sim E^{-2.7}$, as their parents. The positrons will thereafter suffer radiative losses of energy that will steepen their spectrum, and at energies beyond $\sim 300 \mathrm{GeV}$ their spectrum reaches an asymptotic form $\sim E^{-3.7}$ i.e. with an index steeper by one unit compared with that of the protons $[8,12]$. We may refer to Fig. 5 to see that the spectrum of positrons is smooth and is nearly identical to that of the primary nucleonic component that generates it. The complex behavior of the observed $e^{+} /\left(e^{+}+e^{-}\right)$ratio is attributable to the evolving shape of the primary electron spectrum and the steepening of the $e^{+}$spectrum beyond $300 \mathrm{GeV}$. The details are found in our earlier paper [7,8]; as noted earlier the positron fraction is expected to reach a value $\sim 0.6$ at energies beyond $\sim 2 \mathrm{TeV}$, when the primary electrons suffer significant energy losses and are unable to reach the solar system with these energies even from the nearest cosmic ray sources [13].

With a lifetime of $\sim 2.3 \mathrm{Myr}$ up to hundreds of TeV, the expected anisotropies (see Fig. 6) in the cosmic-ray fluxes are small and are consistent with the upper bounds established by the observations[7].

\section{Discussion}

Even though our approach in this paper has been mainly phenomenological, we briefly com- 
ment on the basic assumptions of the NLB model:

1. The injection spectrum $E^{-2.7}$, for the primary cosmic rays, is significantly steeper than the $E^{-2}$ spectrum expected for cosmic rays accelerated in high Mach number plane parallel shocks. However, recent theoretical developments [14] show that steeper spectra even beyond $E^{-3.1}$ can be achieved [15]. In the alternate models which are currently in vogue [16], $\tau_{I S M}(E) \sim E^{-0.3}$, which requires the source spectrum to be $E^{-2.4}$, again steeper than the initial expectation in acceleration by shocks.

2. Can the diffusion constant in the interstellar medium be independent of (or very weakly dependent on) the energy as postulated by the NLB model? It is possible that the diffusion of the flux tubes could lead to a constant diffusion constant. Also if the scattering occurs due to static magnetic inhomogeneities, such as those generated by flux freezing in an inhomogeneous interstellar medium, the guiding centers will randomly move around and can generate diffusion nearly independent of the energy of the particle, without any concomitant diffusion in energy space. The energy dependence of the diffusion constant in the range of energies of interest may be suppressed by a suitable choice of the spectrum of these magnetic inhomogeneities.

\section{Alternate models and future work}

In the alternate scenario where scattering exclusively by Alfven waves is postulated, the spatial diffusion is accompanied by diffusion in energy space. In fact, this diffusion in energy is evoked in these models [16] to fit the B/C ratio. Since all particles diffusing in ISM through scattering of Alfven waves suffer a modification in their spectra, because of the diffusion in energy space, the injection of the primary particles should not be a power law, but one that after reacceleration is the smooth shape that is observed in Galactic cosmic rays. Furthermore, these models predict $e^{+}$and $\bar{p}$ spectra very different from the smooth spectra which have an index of $\sim 2.7$. Thus the alternate astrophysical or dark matter sources of $\bar{p}$ and $e^{+}$should put out such spectra, which after energy dependent transport and reacceleration add up with the secondary component to match the observed $e^{+}$and $\bar{p}$ spectra, an unlikely coincidence. Keeping these comments in mind, we feel that the NLB model offers a plausible paradigm and it is important that further work should focus on placing it in a proper astrophysical setting and on firm theoretical grounds.

\section{Summary}

We note that production of secondary particles (boron, antiprotons, and positrons) in the NLB model can explain not only the observations of the B/C ratio, which decreases with increasing energy up to a few hundred $\mathrm{GeV} /$ nucleon, but also the antiproton/proton and positron/proton ratios, which in contrast are observed to be essentially energy-independent from $10 \mathrm{GeV}$ to a few hundred $\mathrm{GeV}$. In the model currently in use [16] and in fact in any model in which the secondary boron, antiprotons, and positrons are all produced in the same region - the general interstellar medium of the Galaxy, the falling B/C ratio implies energy-dependent leakage for all particles, thereby forcing the antiproton/proton, and positron/proton ratios also to fall with energy, contrary to observations. 
On the other hand, the NLB model exploits the differences in the kinematics of the spallation reactions and that of high-energy interactions producing antiprotons and pions to ensure that the energy-dependent part of the $\mathrm{B} / \mathrm{C}$ ratio is generated in a local region surrounding sources, without generating too many positrons or antiprotons. The interactions of cosmic rays in the interstellar medium, where leakage is energy-independent, generate all the secondary particles, $\mathrm{B}, e^{+}$and $\bar{p}$, with energy independent ratios with respect to their parent particles. The NLB model also provides a natural explanation for the low level of anisotropy for the cosmic rays[7].

\section{References}

[1] O. Adriani et al., PRL 102, 051101 (2009); PRL 105, 121101 (2010); J. Wu (PAMELA Coll.): Astrophys. Space Sci. Trans., 7, 225âĂŞ228, (2011).

[2] A. Yamamoto et al., (BESS Coll.): The BESS program, Nucl. Phys. B (Proc. Suppl.), 166, 62âĂŞ67, (2007).

[3] J. Casaus, AMS-02 experiment on the ISS, JPCS, 171 (1), p. 012045, (2009).

[4] M. Aguilar et al., AMS Collaboration, Phys. Rev. Lett. 110, 141102, (2013).

[5] AMS Collaboration announcement, CERN, Geneva, Switzerland, 15 April 2015: âĂIJAMS Days at CERNâĂİ and âĂIJLatest Results from the AMS Instrument on the International Space StationâĂİ.

[6] R. Cowsik and L. W. Wilson, Proc. 14th ICRC, Vol2, p. 659, (1975).

[7] R. Cowsik and B. Burch, Phys. Rev. D, 82, 023009, (2010); XVI International Sympoisium on very high energy interaction, 2010, Batavia, IL, USA, (28 June, 2010); Proc. 31st ICRC, Lodz, Poland, (2009).

[8] R. Cowsik, B. Burch and T. Madziwa-Nussinov, ApJ, 786, 124, doi:10.1088/0004- 637X/786/2/124, (2014).

[9] S. A. Stephens, Astrophys. \& Space Sci. 76, 87 (1981); L. C. Tan and L. K. Ng, J. Phys. G. Nucl. Phys., 9, 227, (1983); T. Gaisser and R. Schaefer, Ap. J. 394, 174, (1992); M. Simon et al., Ap.J 499 250 doi:10.1086/305606, (1998).

[10] M. di Mauro, F. Donato, A. Goudelis, and P. D. Serpico, arXiv:1408.0288; Phys. Rev. D 90, 085017 (2014).

[11] R. Kappl and M. Winkler, arXiv:1408.0299v1, (2014).

[12] R. Cowsik, Yash Pal and S.N. Tandon, Phys. Rev. Lett., 17, 1298 (1966).

[13] R. Cowsik and M.A. Lee, 1979, Ap.J., 228, 297 (1979). J. Nishimura et al. Advances in Space Res. 19, 767, (1997). P. Blasi, Phys. Rev. Lett., 103, 051104 (2009).

[14] Y. Uchiyama et al. Ap.J., Letters 723, L122 (2010); P. Blasi et al., Ap.J 755, 121 (2012).; S.H. Lee et al. arXiv: [504.05313v1] [astroph.HE] 21, April, 2015.

[15] M. Cardillo et al., Astron. Astrophysics 565, A74 (2014).

[16] I. Moskalenko and A. Strong, Ap.J 509, 212228, [astro-ph/9807150], (1998); R. Trotta et al.,; arXiv:0037v3 [astr.ph.HE] 2011; A. W. Strong, I. V. Moskalenko, and V. S. Ptuskin, Annu. Rev. Nucl. Part. Sci.57, 285 (2007).

[17] J. J. Engelmann et al., Astron. Astrophys. 233, 96 (1990). 
[18] R. Dwyer, Astrophys. J. 322, 981 (1987).

[19] J. H. Chapell and W. R. Webber, Proc. ICRC17 Paris, 2, 59, (1981).

[20] A. Obermeier et al., ApJ 742 14, doi:10.1088/0004-637X/742/1/14, (2011).

[21] D. MÃijller et al., Astrophys. J. 374, 356 (1991).

[22] H. S. Ahn et al., Astropart. Phys. 30, 133 (2008).

[23] A. D. Panov et al., Proceeding of 30th ICRC, 3, (2008).

[24] J. Buckley et al., ApJ., 429, 736 (1994).

[25] T. Antoni et al., Astrophys. J. 604, 687 (2004).

[26] R. U. Abbasi et al., arXiv:0907.0498v1.

[27] M. Amenomori et al., Proc. ICRC28, p. 143, (2003).

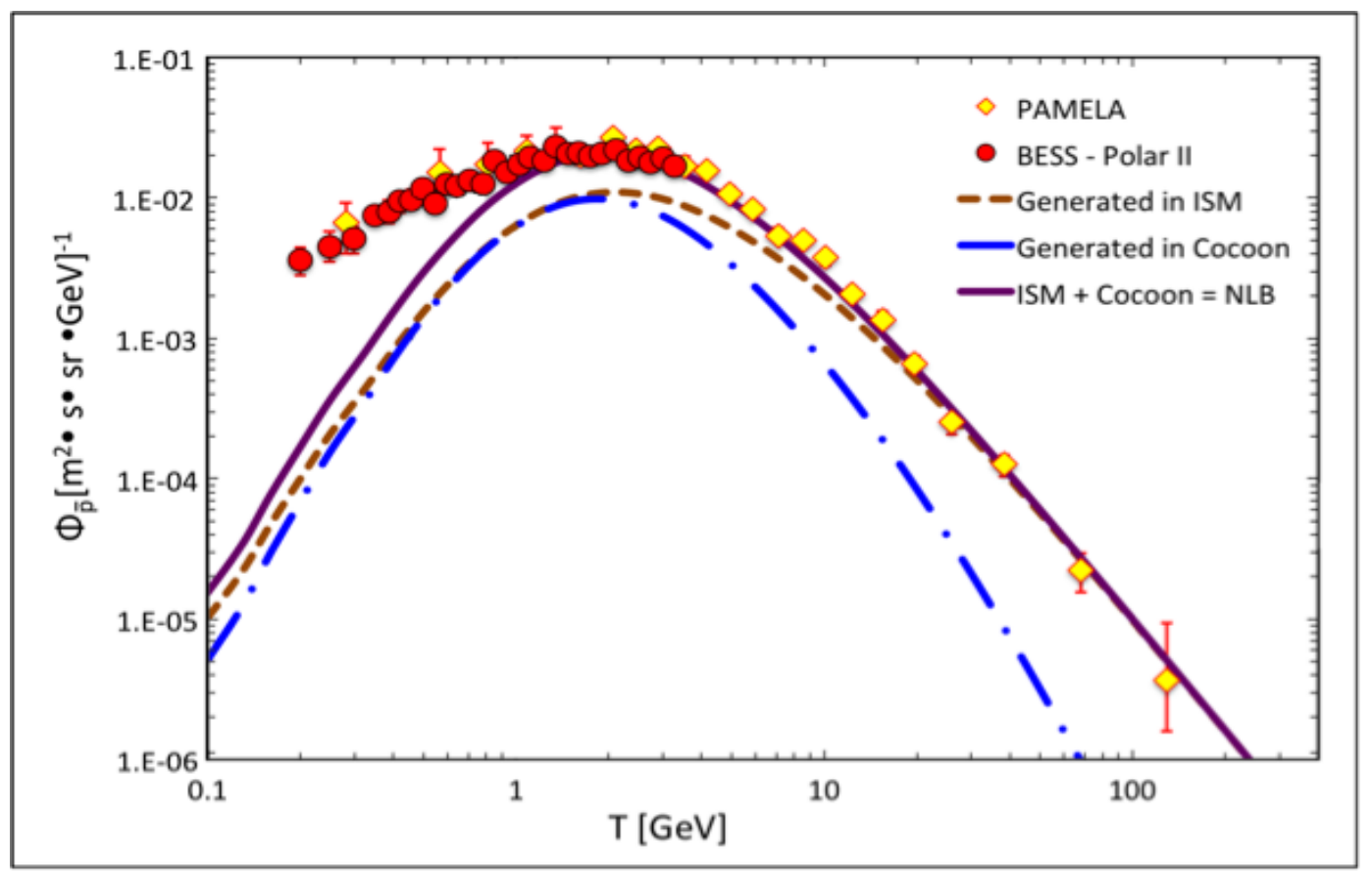

Figure 1: The spectrum of antiprotons observed with the PAMELA and BESS instruments are shown as filled dots [1] and diamonds [2]. In this paper we have interpreted the antiproton spectrum as the sum of two components: (1) that generated in the ISM (red dash-dotted line) where the residence time of cosmic rays is independent of their energy and (2) a small component at energies below $10 \mathrm{GeV}$, with a steep energy dependence at higher energies, generated in a cocoon-like region (blue chain dotted line) surrounding the sources of primary cosmic rays. 


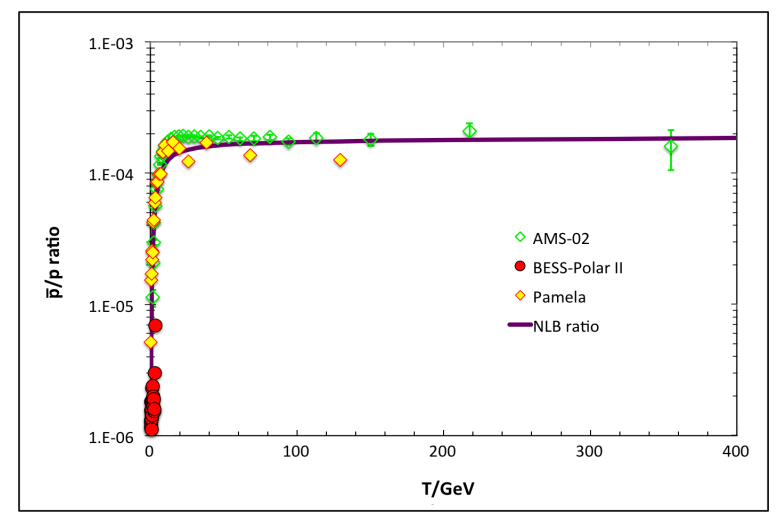

Figure 2: The solid line represents the ratio of the theoretically estimated antiproton flux to the empirical fit to the observed proton flux. The observations from PAMELA, BESS and the AMS-02 instruments [1-5] are shown. The flatness of the $\bar{p} / p$ ratio implies that the leakage lifetime is independent of energy for the Galactic cosmic rays.

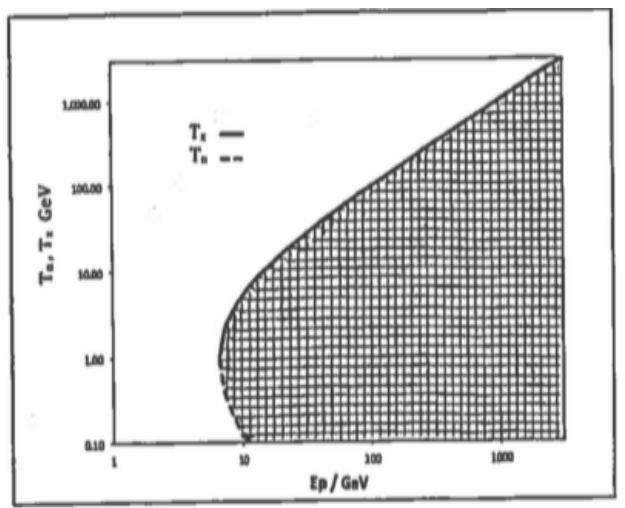

Figure 3: The kinematics of the production of $\bar{p}$ in high-energy collision of cosmic-ray protons is shown. Beyond the threshold at $E_{p} \sim 7 m_{p}, p-b a r$ is produced in the finely gridded region between the maximum kinetic energy of $T_{x}$ and a minimum kinetic energy of $T_{n}$, for any given energy of the primary proton.

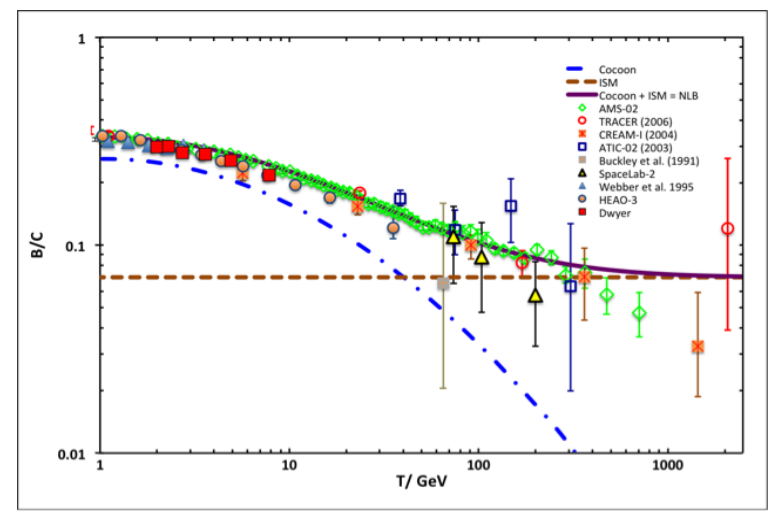

Figure 4: The observed B/C ratio is plotted along with the spectra expected nested leaky-box (NLB) model. The B/C data presented was taken from previous[17-24] and recent AMS experiments [4-5]. 


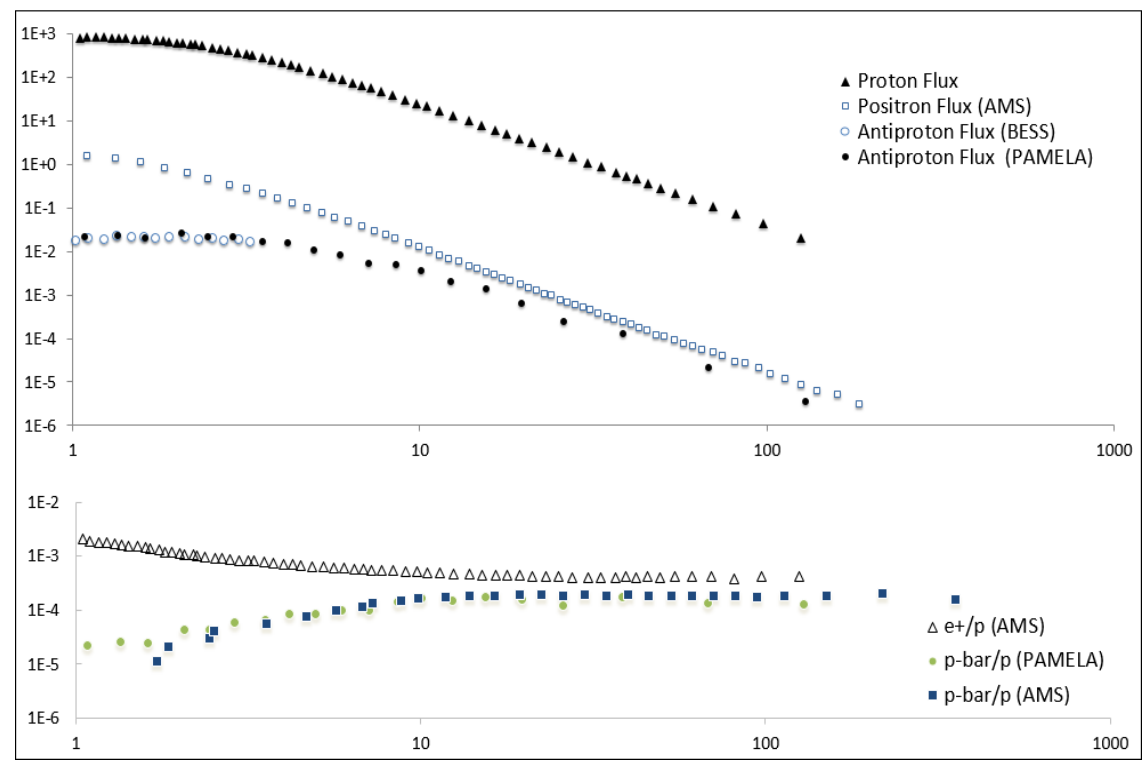

Figure 5: The spectra of $e^{+}$and $\bar{p}$ are compared in the upper panel with that of their progenitors, the primary cosmic ray protons. At energies beyond $\sim 10 \mathrm{GeV}$ they have nearly identical spectral characteristics except for the effects of energy losses suffered by the positrons. In these spectra or in the spectral ratios (lower panel) there is no indication of the presence of multiple components.

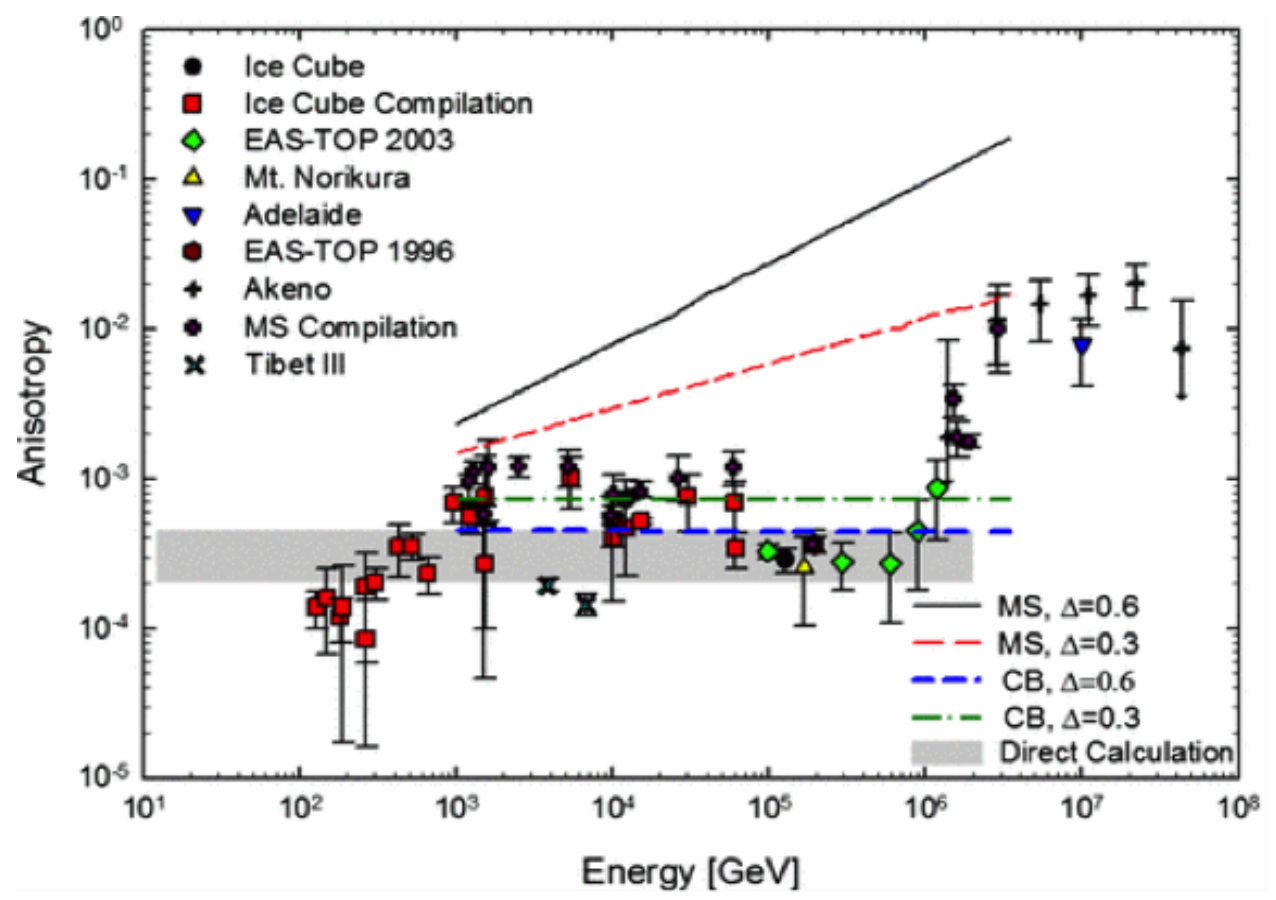

Figure 6: Measurements of the cosmic-ray anisotropy from various compilations [16, 25-27]. Also plotted are the predictions from models in M-S [16] and the results from Eq. (7) of Cowsik and Burch [7], which are labeled as CB. The gray region shows the predicted anisotropy from Eq. (8), of Cowsik and Burch [7]. 